TRANSACTIONS OF THE

AMERICAN MATHEMATICAL SOCIETY

Volume 353, Number 4, Pages 1387-1401

S 0002-9947(00)02777-X

Article electronically published on December 15, 2000

\title{
EFFECTIVE ESTIMATES ON THE VERY AMPLENESS OF THE CANONICAL LINE BUNDLE OF LOCALLY HERMITIAN SYMMETRIC SPACES
}

\author{
SAI-KEE YEUNG
}

\begin{abstract}
We study the problem about the very ampleness of the canonical line bundle of compact locally Hermitian symmetric manifolds of non-compact type. In particular, we show that any sufficiently large unramified covering of such manifolds has very ample canonical line bundle, and give estimates on the size of the covering manifold, which is itself a locally Hermitian symmetric manifold, in terms of geometric data such as injectivity radius or degree of coverings.
\end{abstract}

Let $L$ be an ample line bundle on an algebraic manifold $M$. From Kodaira's Embedding Theorem, we know that $m L$ is very ample if $m$ is sufficiently large so that the sections of $m L$ give rise to an embedding of $M$ into some projective space. A natural question is on the estimates of such $m$ so that $m L$ is very ample. In particular, one may ask the same question for the canonical line bundle of a manifold with negative first Chern class, or of general type. Of particular interest among such manifolds is the class of locally Hermitian symmetric spaces. The main purpose of this article is to show that for compact locally Hermitian symmetric manifolds of non-compact type, $K$ is very ample if the injectivity radius of the manifold is greater than some effective constant which can be estimated in terms of some geometric data.

Effective estimates for $m K=K+(m-1) K$ with $m \geq 2$ in terms of injectivity radius have been obtained in [HT] and [Y2]. The difficulty in the case of $m=1$ can be seen from the fact that in constructing holomorphic sections from the BochnerKodaira technique, one always need $K+L$ for some extra positivity from $L$. In [Y2], it is shown that for a tower of coverings of compact Hermitian locally symmetric manifolds $M_{i}, K_{M_{i}}$ becomes very ample when $i$ approaches to $\infty$. To compensate for the extra positivity required for the Bochner-Kodaira technique, we prove our result by showing that the limit of the Bergman kernel on $M_{i}$ approaches the corresponding one on the universal covering. However, the limiting process makes the whole approach highly non-effective and requires an infinite sequence of normal coverings of the original manifold. The main purpose of this article is to show the very ampleness of $K_{M}$ for any unramified covering $M$ of $M_{o}$ with injectivity radius or degree of the coverings greater than some effectively estimable constant. For this purpose, an alternate approach using heat kernel estimates combined with Atiyah's

Received by the editors April 10, 2000.

2000 Mathematics Subject Classification. Primary 14E25, 32J27, 32Q05, 32Q40.

Key words and phrases. Very ampleness, canonical embedding.

The author was partially supported by grants from the National Science Foundation.

(C)2000 American Mathematical Society 
Covering Index Theorem is taken to bypass the limiting process. The main difficulty lies in the uniform control of the trace of all the global holomorphic sections and its derivatives at each point of the manifold. This delicate point explains the length of the arguments in Section 3. The main results are stated as Theorem 2 in Section 3 , and Theorems 3 and 4 in Section 4.

The arguments of this paper work for other algebraic manifolds whose universal covering admits a lot of holomorphic functions so that the Bergman kernel is well behaved. Apart from locally Hermitian symmetric manifolds, the arguments in particular can be applied to those Kähler manifolds whose Riemannian sectional curvature is bounded between two negative constants.

The author would like to thank Ngaiming Mok, Yum-Tong Siu and Wing-Keung To for helpful discussions in the preparation of this article.

\section{Preliminaries}

Let $V^{i}=\wedge^{i} T_{M}^{*}$ be the space of exterior differential forms on $M$. Let $\Delta_{i}$ be the Laplacian acting on $V^{i}$. We have $\Delta_{i}=d d^{*}+d d^{*}$, where $d: \wedge^{i} T^{*} \rightarrow \wedge^{i+1}$ is given by

$$
d\left(\sum_{j_{1}, \ldots, j_{i}} f_{j_{1} \ldots j_{i}} d x^{j_{i}} \wedge \cdots \wedge d x^{j_{i}}\right)=\sum_{j, j_{1}, \ldots, j_{i}} \frac{\partial f_{j_{1} \ldots j_{i}}}{\partial x_{j}} d x^{j} \wedge d x^{j_{i}} \wedge \cdots \wedge d x^{j_{i}}
$$

and $d^{*}$ is the formal adjoint of $d$.

As the principal symbol of $\Delta_{i}$ is a scalar multiple in the sense that $\sigma_{\Delta_{i}}(v, v) s=$ $-g(v, v) s$ for any local exterior $i$-form $s$ and $v \in T^{*} M$, the usual construction of heat kernel can be applied to the heat equation $\left(\frac{\partial}{\partial t}+\Delta_{i}\right) k_{i}(t, x, y)=0$. The heat kernel $k_{i}(t, x, y)$ is defined to be $\operatorname{Hom}\left(V_{x}^{i}, V_{y}^{i}\right)$-valued functions on $(0, \infty) \times \tilde{M} \times \tilde{M}$ satisfying the above heat equation and other properties similar to those in $([\mathbb{P}$, page 241) and ([Do1], page 489). As the metric $h$ on $V$ induces an isomorphism of the dual $V^{i *}$ of $V$ to $V$, we may identify $\operatorname{Hom}\left(V_{x}^{i}, V_{y}^{i}\right)$ with $V_{x}^{i} \otimes V_{y}^{i}$. For $x=y$, we can then define the trace of a homomorphism in $\operatorname{Hom}\left(V_{x}^{i}, V_{x}^{i}\right)$ by taking the inner product with respect to the metric $h$, which is the same as the trace of the homomorphism with respect to an orthonormal basis at $x$. The trace of the heat kernel $\operatorname{Trk}_{i}(t, x, x)$ is then defined and is the same as its magnitude with respect to the metric involved.

Lemma 1. Let $\tilde{M}$ be a complete manifold which is the universal covering of a compact manifold $M$. Consider the heat kernel $\tilde{k}^{i}$ of the laplacian $\Delta_{i}$ obtained with the lift of the metric from $M$. Let $T>0$ be an arbitrary fixed number. Then the heat kernel on $\tilde{M}$ satisfies the following estimates for $T \geq t \geq 1$ :

$$
\left\|\tilde{k}_{i}(t, x, y)\right\| \leq c_{1} \exp \left[-\frac{d^{2}(x, y)}{4 t}\right],
$$

where $c_{1}$ is a constant depending only on $T$.

Proof. This follows from the standard construction of $[\mathrm{P},[\mathrm{BGM}]$ and especially Do1.

Lemma 2. Let $\tilde{k}_{i}(t, x, y)$ be the heat kernel of the Laplacian $\Delta_{i}$ on $i$-forms of a Riemannian manifold $\tilde{M}$ which covers a compact manifold. Let $\tilde{\mathcal{H}}_{i}$ be the space of harmonic $i$-forms with $L^{2}$-norm 1 on $M$. Let $\phi_{j}$ be an orthonormal basis for $\tilde{\mathcal{H}}_{i}$. Let $\tilde{H}_{i}(x, y)=\sum_{\phi_{j} \in \tilde{\mathcal{H}}_{i}} \phi_{j}(x) \otimes \phi_{j}(y)$. Then, given any $\delta>0$, there is a computable 
constant $r_{2}$ depending only on $\tilde{M}$ such that for all $t>r_{2}$ and all points $x \in \tilde{M}$, $\left|\operatorname{Tr} \tilde{k}_{i}(t, x, x)-\operatorname{Tr} \tilde{H}_{i}(x, x)\right|<\delta$.

Proof. First we note that as $\tilde{M}$ covers a compact manifold $M$, and the heat kernel is invariant under isometry, $k_{i}(t, x, x)$ is bounded for all $t \geq 1$. From spectral decomposition we get

$$
k_{i}(t, x, y)=\int_{\sigma\left(\Delta_{i}\right)} e^{-\lambda t} d E_{x, y}^{i}(\lambda),
$$

where $\sigma\left(\Delta_{i}\right)$ is the spectrum of $\Delta_{i}$ on $\tilde{M}$, and the spectral measure $d E_{x, y}^{i}(\lambda)$ is the spectral projection valued measure from $y$ to $x$. Hence

$$
\operatorname{Tr}_{i}(t, x, x)=\int_{\sigma\left(\Delta_{i}\right)} e^{-\lambda t} d E_{x, x}^{i}(\lambda)=\operatorname{Tr} \tilde{H}_{i}(x, x)+\int_{\sigma\left(\Delta_{i}\right) \cap(0, \infty)} e^{-\lambda t} d E_{x, x}(\lambda) .
$$

For $\epsilon>0$, define $F(\epsilon, t)=\int_{\sigma\left(\Delta_{i}\right) \cap(0, \epsilon)} e^{-\lambda t} d E_{x, x}(\lambda)$. Note that $F(\epsilon, 1)$ is a monotonic increasing function in $\epsilon$ and $\lim _{\epsilon \rightarrow 0} F(\epsilon, 1)=0$ as the domain of integration approaches to an empty set. In fact it is estimated by [Lü] that $F(1, \epsilon) \leq c \frac{1}{-\log (\epsilon)}$ for some controllable constant $c>0$.

Now given any $\delta>0$, we can find an $\epsilon>0$ such that

$$
F(\epsilon, 1)=\int_{\sigma\left(\Delta_{i}\right) \cap(0, \epsilon)} e^{-\lambda} d E_{x, x}(\lambda)<\frac{\delta}{2} .
$$

$\lambda \geq \epsilon$ implies that $e^{-\lambda t} \leq e^{-\frac{\epsilon}{2} t} e^{-\frac{\lambda}{2} t}$, and hence

$$
\int_{\sigma\left(\Delta_{i}\right) \cap[\epsilon, \infty)} e^{-\lambda t} d E_{x, x}(\lambda) \leq e^{-\frac{\epsilon}{2} t} \operatorname{Tr} k_{i}\left(\frac{t}{2}, x, x\right) \leq e^{-\frac{\epsilon}{2} t} C .
$$

If $t$ is chosen to be so large that $e^{-\frac{\epsilon}{2} t}<\frac{\delta}{2}$, we conclude that

$$
\begin{aligned}
& \left|\operatorname{Tr} \tilde{k}_{i}(t, x, x)-\operatorname{Tr} \tilde{H}_{i}(x, x)\right| \\
& \quad=\int_{\sigma\left(\Delta_{i}\right) \cap(0, \epsilon)} e^{-\lambda} d E_{x, x}(\lambda)+\int_{\sigma\left(\Delta_{i}\right) \cap[\epsilon, \infty)} e^{-\lambda t} d E_{x, x}(\lambda)<\delta .
\end{aligned}
$$

Lemma 3. Let $\tilde{M}$ be the universal cover of a compact manifold $M$ whose sectional curvature is bounded from below by $-b$ and the eigenvalues of the curvature operator are bounded from above by $b_{1}$. Let $T>1$ be a fixed number. There exists a constant $c=c(T)$ such that if the injectivity radius $\tau=\tau(M)$ of $M$ satisfies $\tau>c$, then for all $0 \leq t \leq T$ and $x \in \tilde{M}$

$$
\left|\operatorname{Tr} k_{i}(t, x, x)-\operatorname{Tr} \tilde{k}_{i}(t, x, x)\right| \leq \exp \left(-\frac{\tau^{2}}{12 t}\right)
$$

for all points $x \in M$. Here $c(T)$ depends only on the curvature bounds.

Proof. The idea is similar to the arguments in [Do1]. It essentially follows from the fact that

$$
k_{i}(t, x, x)=\sum_{\gamma \in \Gamma} \tilde{k}(t, x, \gamma x)=\tilde{k}(t, x, x)+\sum_{\gamma \in \Gamma-\{1\}} \tilde{k}(t, x, \gamma x)
$$

in the sense of uniform convergence, and estimates of the second term above from Lemma 1. 


\section{Effective estimates on the Bergman Kernel}

Proposition 1. Assume that the $L^{2}$ cohomology group of the universal covering $\tilde{M}$ of $M$ satisfies $\tilde{h}_{i}(\tilde{M})=0$ for $0 \leq i \leq n-1$, where $n$ is the complex dimension of $M$. Then, given any $\epsilon>0$, there exists $r=r(\epsilon)$, depending on $\tilde{M}$, such that the estimates

$$
\left|\operatorname{Tr} H_{i}(x, x)\right| \leq \epsilon
$$

hold for all $x \in M$ if the injectivity radius satisfies $\tau>r$.

Proof. Let $T=r_{1}$ be the constant given by Lemma 2, so that for $t \geq T$

$$
\left|\operatorname{Tr} \tilde{k}_{i}(t, x, x)-\operatorname{Tr} \tilde{H}_{i}(x, x)\right| \leq \delta .
$$

Let $c(T)$ be the constant in Lemma 3. Choose $r_{2}(T)=\max (c(T), \sqrt{-12 T(\log \delta)}+1)$ so that $\exp \left(-\frac{(i \tau)^{2}}{12 T}\right)<\delta$ and hence $\left|k_{i}(T, x, x)-\tilde{k}_{i}(T, x, x)\right|<\delta$ for $\tau>r_{2}(T)$. Let $r_{3}(T)=\max \left(T, c(T), r_{2}(T)\right)$. Then for $\tau>r_{3}(T)$, we conclude that

$$
\begin{aligned}
0 & \leq \operatorname{Tr} H_{i}(x, x) \leq \operatorname{Tr} k_{i}\left(r_{3}, x, x\right) \\
& \leq\left|\operatorname{Tr} k_{i}\left(r_{3}, x, x\right)-\operatorname{Tr} \tilde{k}_{i}\left(r_{3}, x, x\right)\right|+\left|\operatorname{Tr} \tilde{k}_{i}\left(r_{3}, x, x\right)-\operatorname{Tr} \tilde{H}_{i}(x, x)\right|+\operatorname{Tr} \tilde{H}_{i}(x, x) \\
& \leq 2 \delta
\end{aligned}
$$

This concludes the proof of the proposition (we choose $\epsilon=2 \delta$ ).

Theorem 1. Assume that the $L^{2}$ cohomology group of the universal covering $\tilde{M}$ of $M$ satisfies $\tilde{h}_{i}(\tilde{M})=0$ for $0 \leq i \leq n-1$. Suppose $M$ is a holomorphic covering of $M_{o}$. Assume that $\operatorname{vol}\left(M_{o}\right)=V_{o}$ is given. Given any $\alpha>0$, there is a constant $r$, depending only on $\tilde{M}$ and $V_{o}$, such that

$$
\left|\operatorname{Tr} H_{n}(x, x)\right| \geq(1-\alpha)\left|\operatorname{Tr} \tilde{H}_{n}(x, x)\right|
$$

for all $x \in M$ if the injectivity radius satisfies $\tau>r$.

Proof. Consider the Euler characteristics $\chi(M)=\sum_{i=0}^{n} h^{i}(M)$ on $M$ and the $L^{2}$ analogue on the universal covering $\tilde{M}, \chi^{(2)}(\tilde{M})=\sum_{i=0}^{n} \tilde{h}^{i}(\tilde{M})$. Atiyah's Covering Index Theorem $\left[\mathrm{A}\right.$ implies that $\chi(M)=\chi^{(2)}(\tilde{M})$, and hence

$$
\tilde{h}^{n}(\tilde{M})-h^{n}(M)=\sum_{i=0}^{n-1} h^{i}(M) .
$$

Recall that $h^{i}(M)=\int_{M} \operatorname{Tr} H_{i}(x, x)$ and $\tilde{h}^{i}(\tilde{M})=\int_{M} \operatorname{Tr} \tilde{H}_{i}(x, x)$, where the second integral is taken over a fundamental domain of $M$ on $\tilde{M}$. Since the Galois transformation group on $M$ corresponding to the covering map of $M$ to $M_{o}$ is a biholomorphism and the Schwarz kernels are invariant under biholomorphism, this can also be rewritten as

$$
\int_{M_{o}}\left(\operatorname{Tr} \tilde{H}^{n}(\tilde{M})-\operatorname{Tr} H^{n}(M)\right)=\sum_{i=0}^{n-1} \int_{M_{o}} \operatorname{Tr} H^{i}(M) .
$$

On the other hand, it follows from the previous proposition that

$$
\left|\sum_{i=0}^{n-1} \int_{M_{o}} \operatorname{Tr} H^{i}(M)\right| \leq\left[\frac{n}{2}\right] \epsilon V_{o}
$$


if $\tau(M) \geq r(\epsilon)$. We also note that $\tilde{H}^{n}(\tilde{M})(x, x)$ is invariant under biholomorphism on $\tilde{M}$, and hence is a contant function.

Let $x \in M$ be any point. Without loss of generality, we may assume that the injectivity radius of $M, \tau(M) \geq 1$. Let $\sigma<1$. Let $B_{\sigma}(x)$ be a ball of radius $\sigma$ around $x$. Consider a complex geodesic coordinate in a normal neighbourhood of $x$. In terms of the Euclidean coordinates, consider the Euclidean metric $g^{E}$, which agrees with our metric $g$ up to second order at $x$, so that $g^{E}(x)=g(x)+\mathcal{O}\left(|x|^{2}\right)$. There exist two Euclidean balls of optimal radius $r_{1}^{E}(\sigma)$ and $r_{2}^{E}(\sigma)$ respectively so that $B_{r_{1}(\sigma)}^{E}(x) \subset B_{\sigma}(x) \subset B_{r_{2}(\sigma)}^{E}(x)$, and as $\sigma<1$, we can find absolute constants $a_{1}, a_{2}$ so that

$$
a_{1} \operatorname{vol}^{E}\left(B_{r_{1}(\sigma)}^{E}(x)\right) \leq \operatorname{vol}^{g}\left(B_{\sigma}(x)\right) \leq a_{2} \operatorname{vol}^{E}\left(B_{r_{1}(\sigma)}^{E}(x)\right),
$$

the constants depending only on the curvature bound.

Recall that

$$
H^{n}(M)(x, x)=\sum_{i=1}^{N}\left\|s_{i}\right\|^{2}=\sum_{i=1}^{N}\left|f_{i}(x)\right|^{2} h,
$$

where $s_{i}=f_{i} d z^{1} \wedge \cdots \wedge d z^{n}$ is an orthonormal bases of holomorphic $n$-forms, and $h$ is the metric of the canonical line bundle. We have $0<e_{1}<h<e_{2}$ for some constants $e_{1}, e_{2}$ on $B_{\sigma}(x)$ depending only on the curvature bounds of $M$. From our earlier lemma, we conclude easily that

$$
H^{n}(M)(x, x) \leq \tilde{H}^{n}(\tilde{M})(x, x)=c
$$

for all $x$. Hence we conclude that $\sum_{i=1}^{N}\left|f_{i}(x)\right|^{2} \leq \frac{c}{e_{1}}$. From Cauchy's integral formula we get

$$
f^{\prime}(x)=\int_{\partial \sigma(x)} \frac{f(s)}{s-x} d s
$$

and hence

$$
\begin{aligned}
\left.\left|\sum_{i=1}^{N}\right| f_{i}^{\prime}(x) f_{i}(x)\right|^{2} & =\left|\int_{\partial \sigma(x)} \sum_{i} \frac{f_{i}(s) f_{i}(x)}{s-x} d s\right| \\
& \leq \int_{\partial \sigma(x)} \sum_{i} \frac{\left|f_{i}(s)\right|^{2}+\left|f_{i}(x)\right|^{2}}{2 \sigma} d s \\
& \leq \frac{2 \pi c}{e_{1}} .
\end{aligned}
$$

It follows that the Euclidean derivative of $\sum_{i=1}^{N}\left|f_{i}(y)\right|^{2}$ is bounded by $\frac{4 \pi c}{e_{1}}$ for all $y \in B_{\sigma}(x)$, and hence, by direction integration from $x$ to $y$ along a real straight line segment,

$$
\sum_{i=1}^{N}\left|f_{i}(y)\right|^{2} \leq \sum_{i=1}^{N}\left|f_{i}(x)\right|^{2}+\frac{4 \pi c}{e_{1}}|y-x|
$$


Suppose now that $\left\|H^{n}(M)(x, x)\right\| \leq \eta$. Then

$$
\begin{aligned}
& \int_{B_{\sigma}(x)}\left|H^{n}(M)(y, y)\right|_{g} d v_{g} \\
= & \int_{B_{\sigma}(x)}\left|H^{n}(M)(y, y)\right|_{g^{E}} d v_{g^{E}} \\
= & \int_{B_{\sigma}(x)} \sum_{i=1}^{N}\left|f_{i}(y)\right|^{2} d v_{g^{E}} \\
\leq & \int_{B_{\sigma}(x)} \sum_{i=1}^{N}\left[\left|f_{i}(x)\right|^{2}+\frac{4 \pi c e_{2}}{(n+1) e_{1}} r_{2}\right] d v_{g^{E}} \\
= & {\left[\eta+\frac{4 \pi c e_{2}}{(n+1) a_{1} e_{1}} r_{2}\right] \operatorname{vol}\left(B_{\sigma}\right) }
\end{aligned}
$$

We conclude that

$$
\begin{aligned}
\int_{M_{o}}\left|H^{n}(M)(y, y)\right|_{g} d v_{g} & =\int_{M_{o}-B_{\sigma(x)}} H^{n}(M)(y, y)+\int_{B_{\sigma(x)}} H^{n}(M)(y, y) \\
& \leq\left(V_{o}-\operatorname{vol}\left(B_{\sigma(x)}\right)\right) c+\left[\eta+\frac{4 \pi c e_{2}}{(n+1) a_{1} e_{1}} r_{2}\right] \operatorname{vol}\left(B_{\sigma}\right) .
\end{aligned}
$$

On the other hand, by our previous constructions, we have

$$
\int_{M_{o}}\left|H^{n}(M)(y, y)\right|_{g} \geq V_{o} c-\epsilon
$$

Hence

$$
\eta \geq c-\left[\frac{4 \pi c e_{2}}{(n+1) a_{1} e_{1}} r_{2}\right]-\frac{\epsilon}{\operatorname{vol}\left(B_{\sigma}\right)} .
$$

Recall that $r_{2}=r_{2}(\sigma)$ is the the radius of the smallest Euclidean ball at $x$ containing $B_{\sigma}(x)$. Choose $\sigma$ and hence $r_{2}$ so small that $c-\left[\frac{4 \pi c e_{2}}{(n+1) a_{1} e_{1}} r_{2}\right] \geq\left(1-\frac{\alpha}{2}\right) c$. Then choose $\epsilon$ so small that $\frac{\epsilon}{\operatorname{vol}\left(B_{\sigma}\right)}<\frac{\alpha}{2} c$. Now from the previous lemma, we can find $\epsilon$ satisfying the latter condition if the injectivity radius $\tau$ of $M$ is sufficiently large. This concludes the proof of the theorem.

\section{EFFECTIVE VERY AMPLENESS}

First we prove the following lemma.

Lemma 4. In the same notation as in Theorem 1, assume that the Bergman kernels $H^{n}(x, y)$ of $M$ and $\tilde{H}^{n}(x, y)$ of $\tilde{M}$ satisfy $\left\|\tilde{H}^{n}(x, x)-H^{n}(x, x)\right\|<\epsilon$. Then

$$
\left\|\tilde{H}^{n}(x, y)-H^{n}(x, y)\right\|<4 \epsilon
$$

for all $(x, y) \in M$ with the distance $d(x, y)<\frac{\tau}{2}$ if the injectivity radius $\tau$ is greater than an effective constant.

Proof. First we note that, pulling back to the universal covering $\tilde{M}$, which is a bounded domain, the addition or subtraction of the heat and Bergman kernels involved in the following discussions makes sense, since only line bundle is involved and it can be trivialized by the standard canonical section on $C^{n}$. 
Similarly to our arguments of Proposition 1, we consider

$$
\begin{aligned}
& \left|H_{n}(x, y)-\tilde{H}_{n}(x, y)\right| \\
\leq & \left|H_{n}(x, y)-k_{n}(t, x, y)\right|+\left|k_{n}(t, x, y)-\tilde{k}_{n}(t, x, y)\right|+\left|\tilde{k}_{n}(t, x, y)-\tilde{H}_{n}(x, y)\right|
\end{aligned}
$$

Let us begin with the second term $\left|k_{n}(t, x, y)-\tilde{k}_{n}(t, x, y)\right|$, where

$$
k_{n}(t, x, y)=\tilde{k}_{n}(t, x, y)+\sum_{\gamma \in \Gamma-\{1\}} \tilde{k}_{n}(t, x, \gamma y) .
$$

From the assumption that $d(x, y)<\frac{\tau}{2}$, we know that $d(x, \gamma y)>\frac{\tau}{2}$ for all $\gamma \in \Gamma-\{1\}$. Hence, as in Lemma 3,

$$
\left|\operatorname{Tr} k_{i}(t, x, y)-\operatorname{Tr} \tilde{k}_{i}(t, x, x)\right| \leq \exp \left(-\frac{\tau^{2}}{48 t}\right) .
$$

For the third term, given $\delta>0$, the argument of Lemma 2 gives

$$
\left|\tilde{k}_{n}(t, x, y)-\tilde{H}_{n}(x, y)\right|<\delta
$$

for $t>r_{2}$, the notation being the same as in Lemma 2 .

Hence, as in the proof of Proposition 1, for

$$
\tau>r_{3}(T)=\max (T, c(T), \sqrt{-48 T \log \delta}+1)
$$

and $T$ determined by $r_{1}$ in Lemma 2 , we conclude that

$$
\left|k_{n}(t, x, y)-\tilde{k}_{n}(t, x, y)\right|+\left|\tilde{k}_{n}(t, x, y)-\tilde{H}_{n}(x, y)\right| \leq 2 \delta .
$$

We choose $\delta=\epsilon$.

For the first term, in terms of an orthonormal set of eigenvectors,

$$
\begin{aligned}
& \left|k_{n}(t, x, y)-H_{n}(x, y)\right| \\
= & \left|\sum_{\lambda_{i}>0} e^{-\lambda_{i} t} \phi_{i}(x) \phi_{i}(y)\right| \\
\leq & \left|\sum_{\lambda_{i}>0} e^{-\lambda_{i} t} \phi_{i}(x) \phi_{i}(x)\right|^{\frac{1}{2}}\left|\sum_{\lambda_{i}>0} e^{-\lambda_{i} t} \phi_{i}(y) \phi_{i}(y)\right|^{\frac{1}{2}} \\
= & \left|k_{n}(t, x, x)-H_{n}(x, x)\right|^{\frac{1}{2}}\left|k_{n}(t, y, y)-H_{n}(y, y)\right|^{\frac{1}{2}} .
\end{aligned}
$$

But again

$$
\begin{aligned}
& \left|k_{n}(t, x, x)-H_{n}(x, x)\right| \\
\leq & \left|k_{n}(t, x, x)-\tilde{k}_{n}(t, x, x)\right|+\left|\tilde{k}_{n}(t, x, x)-\tilde{H}_{n}(x, x)\right|+\left|\tilde{H}_{n}(x, x)-H_{n}(x, x)\right| \\
\leq & 3 \epsilon,
\end{aligned}
$$

where the last term is estimated by Theorem 1 . Hence $\left|k_{n}(t, x, x)-H_{n}(x, x)\right|^{\frac{1}{2}}<3 \epsilon$ and so $\left|k_{n}(t, x, y)-H_{n}(x, y)\right| \leq 3 \epsilon$.

Together with the estimates for the other two terms, we conclude that

$$
\left|H_{n}(x, y)-\tilde{H}_{n}(x, y)\right| \leq 4 \epsilon .
$$

Proposition 2. In the same notation as before, let $M$ cover $M_{o}$, whose diameter is bounded from above by $d_{o}$. Then there is an effective constant $r$ such that if the radius $r(M)$ of $M$ is larger than $r$ and $x \in M$ is arbitrary, there exists a canonical section $s(x)$ of $K_{M}$ such that $\nabla s \neq 0$. 
Proof. By homogeneity of $\tilde{M}$, we may assume that $x$ is the origin and identify $M_{o}$ with a fundamental domain on $\tilde{M}$ containing 0 . Pulling back to $\tilde{M}$, a section $s$ on $M$ is a holomorphic section of $\tilde{M}$, locally but not globally $L^{2}$. Let $W$ be a vector of unit length on $\tilde{M}$, which is realized as a bounded domain on $C^{n}$. We use $\frac{\partial}{\partial w}$ or $\partial_{W}$ to denote differentiation on $\tilde{M} \subset C^{n}$, and $C_{W, x}$ to denote the complex line through $x$ in the direction of $W$.

Let $h(x, y)=\sum_{i} f_{i}(x) \overline{f_{j}(y)}$ and similarly define $\tilde{h}(x, y)$ for the coefficients of the Bergman kernels on $M$ and $\tilde{M}$. We define

$$
D_{W} h(x, y)=\sum_{i=1}^{N} \frac{\partial f}{\partial w}(x) \frac{\partial f}{\partial w}(y), \quad D_{W} \tilde{h}(x, x)=\sum_{i=1}^{\infty} \frac{\partial \tilde{f}}{\partial w}(x) \frac{\partial \tilde{f}}{\partial w}(y) .
$$

As in the proof of the previous lemma, it suffices for us to do the estimates on a fundamental domain of $M_{o}$ on $\tilde{M}$ and consider the lower bound for $D h(x, x)$. We are going to show that $D h$ differs at most by the order of $\epsilon$ from $D \tilde{h}(x, x)$.

From the holomorphicity and antiholomorphicity of $h(x, y)$ and $\tilde{h}(x, y)$ in $x$ and $y$ respectively, we have the integral representation

$$
\begin{aligned}
& \left|D_{W} h(x, y)\right| \\
& =\left|\sum_{i} \int_{s \in \partial B_{r} \cap C_{W, x}} \int_{t \in \partial B_{r} \cap C_{W, y}}\left[\frac{f_{i}(s)-f_{i}(x)}{s-x}\right]\left[\frac{\overline{f_{i}(t)-f_{i}(y)}}{t-y}\right]\right| \\
& =\mid \sum_{i} \int_{s \in \partial B_{r} \cap C_{W, x}} \int_{t \in \partial B_{r} \cap C_{W, y}}\left[\frac{f_{i}(s) \overline{f_{i}(t)}-f_{i}(x) \overline{f_{i}(t)}-f_{i}(s) \overline{f_{i}(y)}+f_{i}(x) \overline{f_{i}(y)}}{(r-x) \overline{(s-y)}}\right] \\
& =\frac{1}{r^{2}} \int_{s \in \partial B_{r} \cap C_{W, x}} \int_{t \in \partial B_{r} \cap C_{W, y}}|h(s, t)-h(x, t)-h(t, y)+h(x, y)| \\
& \geq \frac{1}{r^{2}} \int_{s \in \partial B_{r} \cap C_{W, x}} \int_{t \in \partial B_{r} \cap C_{W, y}}[|\tilde{h}(s, t)-\tilde{h}(x, t)-\tilde{h}(t, y)+\tilde{h}(x, y)|-4 c \epsilon] \\
& =\left|D_{W} \tilde{h}(x, y)\right|-4 c \epsilon .
\end{aligned}
$$

Here Lemma 4 was used. In particular,

$$
D_{W} h(x, x) \geq D_{W} \tilde{h}(x, x)-4 c \epsilon .
$$

Suppose $W=\sum_{\alpha} w^{\alpha} \frac{\partial}{\partial w^{\alpha}}$ in terms of local coordinates $w^{\alpha}, 1 \leq \alpha \leq n$. We define a section

$$
s(x)=\frac{1}{\int_{\tilde{M}}\left|\bar{w}^{\alpha} z^{\alpha} d V\right|^{2}}\left[\sum_{\alpha} \bar{w}^{\alpha} z^{\alpha} d V\right]
$$

on $\tilde{M}$. Then $\frac{\partial}{\partial w} f=\frac{1}{\operatorname{Vol}^{E}(\tilde{M})}$ is bounded from below, as $\tilde{M}$ is a bounded domain in $C^{n}$. This concludes the proof of the lemma.

Remark. For a complete manifold $\tilde{M}$ with injectivity radius bounded from below, we can always get a lower bound on $D_{W} \tilde{h}$ by considering appropriate $L^{2}$ sections using $L^{2}$-estimates as in [Y2].

Now we state the main theorem.

Theorem 2. Let $\tilde{M}$ be an Hermitian symmetric space and $M$ a cocompact quotient. Assume that there exists a holomorphic action of a finite group on $M$ whose 
quotient is $M_{o}$. Then the canonical line bundle $K_{M}$ of $M$ is very ample if the injectivity radius of $M$ is bounded from below by an effective constant $r$. The effective constant $r$ depends on the following data.

(i) The rate of the convergence of the heat kernel $k_{i}(t, x, y)$ to the Bergman kernel $\mathcal{H}(x, x)$ as $t \rightarrow \infty$. It would be sufficient to estimate $\epsilon$ such that

$$
\int_{\sigma\left(\Delta_{i}\right) \cap(0, \epsilon)} e^{-\lambda} d E_{x, x}(\lambda)<1
$$

as estimated in Lemma 2.

(ii) The upper bound of the diameter of $M_{o}$.

Proof. a. Base point freeness. This follows from Theorem 1, since the existence of a positive lower bound of the Bergman kernel on $M$ implies the non-vanishing of a certain canonical section at every point on $M$.

$b$. Immersion. Let $\nabla$ be the Hermitian connection on the canonical line bundle. We need to show that for each point $x \in M$, and vector $W$, there exist sections $s_{i}$ and $s_{j}$ so that

$$
\nabla_{W}\left(\frac{s_{i}}{s_{j}}\right)(x)=\frac{s_{j} \nabla_{W} s_{i}-s_{j} \nabla_{W} s_{i}}{s_{j}^{2}}(x) \neq 0 .
$$

Since the Bergman metric is defined independently of choice of a basis, we may assume that $s_{1}$ is chosen among all canonical sections of $M$ to take the supremum norm at $x$, i.e.

$$
\left|s_{1}\right|_{g}=\sup _{\|f\|_{g}=1, f \in \Gamma(K)}|f|_{g} .
$$

We then complete the section to an orthonormal basis by adding in sections $s_{2}, \ldots$, $s_{N}$. Let $A_{W}(x)=\sum_{j>2}\left|\nabla_{W} s_{j}(x)\right|_{g}^{2}$. We are going to prove that $A_{W}(x)$ is bounded away from 0 .

Assume on the contrary that $\left|\nabla_{W} s_{j}\right|_{g}=0$ for all $i \geq 2$. It follows from the definition that $s_{j}(x)=0$ for $j \geq 2$. In this way

$$
\begin{aligned}
\left|H^{n}(x, x)\right|_{g} & =\left|s_{1}(x)\right|_{g}^{2}, \\
\partial_{W}\left|H^{n}(x, x)\right|_{g} & =\left(\nabla_{W} s_{1}(x), s_{1}(x)\right)_{g},
\end{aligned}
$$

where $g$ denotes the Hermitian metric on $K$ induced by the original metric $g$ and the second entry is conjugate holomorphic. Applying the same argument to $L^{2}$ canonical sections on $\tilde{M}$, we get

$$
\begin{aligned}
\left|\tilde{H}^{n}(x, x)\right|_{g} & =\left|\tilde{s}_{1}(x)\right|_{g}^{2}, \\
\partial_{W}\left|\tilde{H}^{n}(x, x)\right|_{g} & =\left(\nabla_{W} \tilde{f}_{1}(x), \tilde{s}_{1}(x)\right)_{g} .
\end{aligned}
$$

We note that

$$
0 \leq\left|\tilde{s}_{1}(x)\right|_{g}^{2}-\left|s_{1}(x)\right|_{g}^{2}=\left|\tilde{H}^{n}(x, x)\right|_{g}-\left|H^{n}(x, x)\right|_{g} \leq c \epsilon
$$

with some effective absolute constant $c$. Hence $\left|s_{1}\right|_{g} \geq\left|\tilde{s}_{1}\right|_{g}-c \epsilon$.

As $|\tilde{H}(x, x)|_{g}$ is invariant under a biholomorphism, we get

$$
\left(\nabla_{W} \tilde{s}_{1}(x), \tilde{s}_{1}(x)\right)_{g}=\partial_{W}|\tilde{H}(x, x)|_{g}=0
$$


and hence $\nabla_{W} \tilde{s}_{1}(x)=0$. The arguments of the previous proposition and Cauchy's integral formula imply that

$$
\begin{aligned}
c \epsilon & \geq\left.\left|\partial_{W}\right| \tilde{H}^{n}(x, x)\right|_{g}-\partial_{W}\left|H^{n}(x, x)\right|_{g} \mid \\
& =\left|\sum_{i}\left(\nabla_{W} \tilde{s}_{i}(x), \tilde{s}_{i}(x)\right)_{g}-\sum_{j}\left(\nabla_{W} s_{j}(x), s_{j}(x)\right)_{g}\right| .
\end{aligned}
$$

for some absolute constant $c$. Hence we conclude that $\left|\left(\nabla_{W} s_{1}(x), s_{1}(x)\right)_{g}\right| \leq c \epsilon$, which can be rewritten as $\left|\nabla_{W} s_{1}(x)\right|_{g}\left|s_{1}(x)\right|_{g} \leq c \epsilon$. As a result,

$$
\left|\nabla_{W} s_{1}\right|_{g}^{2} \leq\left(\frac{c \epsilon}{|H(x, x)|_{g}-c \epsilon}\right)^{2}
$$

However, from the previous proposition,

$$
\left|D_{W} H(x, x)\right|_{g} \geq\left|D_{W} \tilde{H}(x, x)\right|_{g}-c \epsilon .
$$

Defining $A_{W}(x)=\left|D_{W} H(x, x)\right|_{g}-\left|\nabla_{W} s_{1}\right|_{g}^{2}$,we conclude that

$$
A_{W}(x) \geq\left|D_{W} \tilde{H}(x, x)\right|_{g}-c \epsilon-\left(\frac{c \epsilon}{\left|H_{W}(x, x)\right|_{g}-c \epsilon}\right)^{2} .
$$

As $\left|D_{W} \tilde{H}(x, x)\right|_{g}$ is bounded uniformly from below on $M_{o}$, it follows that $A_{W}(x)=$ $\sum_{j \geq 2}\left|\nabla_{W} s_{j}(x)\right|_{g}^{2}$ is non-zero for $\epsilon$ sufficiently small. This implies that $\left|\nabla_{W} s_{j}(x)\right|_{g}$ $\neq 0$ for some $j \geq 2$, which implies that the sections of $K$ gives an immersion of $M$. As before, $\epsilon$ depends only on the radius of $M$ and the diameter of $M_{o}$.

c. Separation of points. For this we need some lemmas

Lemma 5. There exists a constant $r_{1}>0$ such that for all $x \in M, \Gamma(M, K)$ separates points on $B_{r_{1}}(x)$.

Proof. Consider the function $A_{W}(x)=\sum_{j \geq 2}\left|\nabla_{W} s_{j}(x)\right|_{g}^{2}$ in the study of immersion of the canonical map, where $s_{1}$ takes the supremum value at $x$ among all canonical sections of $M$ whose $L^{2}$-norm is 1 . Let $s_{1}^{\perp}$ be the space of norm 1 canonical sections in the orthogonal complement of $s_{1}$ with respect to $g$. We have $A_{W}(x)=$ $\sum_{f \in s_{1}^{\perp}}\left|\nabla_{W} f\right|_{g}^{2}$. The estimate on the lower bound of $A_{W}$ in the previous section implies that there is a function $s \in s_{1}^{\perp}$ so that

$$
\left|\nabla_{W} s\right|_{g} \geq C_{1}=\left|D_{W} \tilde{H}(x, x)\right|_{g}-c \epsilon-\left(\frac{c \epsilon}{|H(x, x)|_{g}-c \epsilon}\right)^{2} .
$$

Hence

$$
\left|\nabla_{W}\left(\frac{s}{s_{1}}\right)(x)\right|=\left|\frac{\nabla_{W} s}{s_{1}}(x)\right|=\frac{\left|\nabla_{W} s\right|_{g}(x)}{\sqrt{H}(x, x)} \geq \frac{C_{1}}{\sqrt{H(x, x)}} .
$$

From here on when doing estimates we used the same notation $x$ to denote a point on $M$ and a point of its lift to the universal covering. Consider a small Euclidean ball $B_{r_{1}}^{E}(x)$ around $x$ with $r_{1}$ sufficiently small, to be determined later, assuming for the time being only that $r_{1}<2 \tau=\frac{1-|x|}{2}$. Pulling back to $\tilde{M}$, we can write $s=f d V, s_{1}=f_{1} d V$ so that $f, f_{1}$ are holomorphic functions on $B_{r_{1}}(x)$; the metric $g$ is related to $g_{E}$ by $c_{1} g_{E} \leq g \leq c_{2} g_{E}$. Since $\left\|s_{1}^{2}(x)\right\|=\operatorname{tr} B(x, x)=a^{2}$ at the point $x$, from our choice of $s_{1}$ we conclude that $c_{1} a \leq\left|f_{1}(x)\right| \leq c_{2} a$. For any holomorphic function $f$ with $L^{2}$-norm 1 , the mean value inequality implies that $|f(w)|<C$ for all $w \in B_{2 \tau}^{E}(x)$ for some absolute constant $C$, since the injectivity radius of $M$ is bounded from below by an absolute constant. From this we conclude that $\left|f^{\prime}(w)\right| \leq \frac{C}{\tau}$ for $w \in B_{\tau}^{E}(x)$, from Cauchy type estimates. It follows from direct 
integration that $|f(y)-f(x)| \leq|y-x| \frac{C}{\tau} \leq r_{1} \frac{C}{\tau}$. Using Cauchy's estimates again, we conclude that

$$
\left|\frac{\partial f}{\partial w}(y)-\frac{\partial f}{\partial w}(x)\right| \leq r_{1} \frac{C}{\tau^{2}},
$$

for all $y \in B_{1}^{E}(x)$. Similarly, $\left|\frac{\partial f_{1}}{\partial w}(y)-\frac{\partial f_{1}}{\partial w}(x)\right| \leq r_{1} \frac{C}{\tau^{2}}$. Hence for $y \in B_{r_{1}}^{E}(x)$

$$
\begin{aligned}
& \left|\nabla_{W}\left(\frac{s}{s_{1}}\right)(y)-\nabla_{W}\left(\frac{s}{s_{1}}\right)(x)\right| \\
= & \left|\partial_{W}\left(\frac{f}{f_{1}}\right)(y)-\partial_{W}\left(\frac{f}{f_{1}}\right)(x)\right| \\
= & \left|\left[\frac{\partial_{W} f}{f_{1}^{2}}(y)-\frac{\partial_{W} f}{f_{1}^{2}}(x)\right]-\left[\frac{f \partial_{W} f_{1}}{f_{1}^{2}}(y)-\frac{f \partial_{W} f_{1}}{f_{1}^{2}}(x)\right]\right|
\end{aligned}
$$

As $c_{1} a \leq\left|f_{1}(x)\right| \leq c_{2} a$ and thus $\left|f_{1}(y)\right| \geq c_{1} a-r_{1} \frac{C}{\tau}$, we conclude that

$$
\left|\nabla_{W}\left(\frac{s}{s_{1}}\right)(y)-\nabla_{W}\left(\frac{s}{s_{1}}\right)(x)\right| \leq r_{1} C_{2}
$$

with $C_{2}$ an absolute constant depending only on $\tau$ and $a$, assuming that $c_{1} a-r_{1} \frac{C}{\tau}>$ 0 . Integrating the expression along any geodesic rays from the origin on $B_{r_{1}}(x)$, we conclude that

$$
\begin{aligned}
\left|\frac{s}{s_{1}}(y)\right|= & \left|\int_{0}^{1} \nabla_{W}\left(\frac{s}{s_{1}}\right)(x+t(y-x)) d t\right| y-x\left|+\frac{s}{s_{1}}(x)\right| \\
= & \left|\int_{0}^{1} \nabla_{W}\left(\frac{s}{s_{1}}\right)(x) d t\right| y-x \mid \\
& +\int_{0}^{1}\left[\nabla_{W}\left(\frac{s}{s_{1}}\right)(x+t(y-x))-\nabla_{W}\left(\frac{s}{s_{1}}\right)(x)\right] d t|y-x|+0 \mid \\
\geq & \left|\int_{0}^{1} \nabla_{W}\left(\frac{s}{s_{1}}\right)(x) d t\right| y-x|| \\
& -\int_{0}^{1}\left|\nabla_{W}\left(\frac{s}{s_{1}}\right)(x+t(y-x))-\nabla_{W}\left(\frac{s}{s_{1}}\right)(x)\right| d t|y-x| \\
\geq & \frac{C_{1}}{\sqrt{H}(x, x)}|y-x|-r_{1} C_{2}|y-x| .
\end{aligned}
$$

Choosing

$$
r_{1}=\min \left(\frac{C_{1}}{2 C_{2} \sqrt{H}(x, x)}, \frac{c_{1} a \tau}{2 C}\right),
$$

we conclude that, $\frac{s}{s_{1}}(y) \neq 0=\frac{s}{s_{1}}(x)$ on $B_{r_{1}}(x)$. As $x$ is arbitrary on $M$, this implies the separation of point in each small neighbourhood of uniform size determined by $r_{1}$.

Now we consider the separation of points whose distance apart is at least $r_{1}$.

Lemma 6. Assume that for any $c>0$, there exists a number $\kappa>0$ such that for every pair of points $x, y \in \tilde{M}$ of distance $d(x, y) \geq r_{1}$, there is always a holomorphic section $s \in \Gamma^{(2)}(\tilde{M}, K)$ satisfying $\|s\|_{L^{2}}=1, s(x)=0,\|s(y)\| \geq \kappa$. Then the canonical map of $M$ takes different values at $x$ and $y$ if $d(x, y)>r_{1}$, provided that the injectivity radius of $M$ is at least $r$ for some effective $r$. 
Proof. From the lemma on the estimate of the difference between $H^{n}$ and $\tilde{H}^{n}$, we know that for each $x \in M$, there exists a canonical section $s_{x}$ such that $\left|s_{x}\right|_{g}(x) \geq c_{1}$ for some absolute constant $c_{1}=\frac{1}{2}\left|\tilde{H}^{n}(x, x)\right|_{g}$ which is independent of $x$. From the mean-value inequality, as argued in the earlier proposition, we also have $\left|s_{x}\right|_{g}(x) \leq$ $c_{2}$ for some absolute contant $c_{2}$. For any pair of points $x, y \in M$, we can find a linear combination $s_{x y}=r s_{x}+(1-r) s_{y}$ so that $c_{3} \leq\left|s_{x y}\right|_{g}(x) \leq c_{4}$. For simplicity of notation, we denote $s=s_{x y}$.

Let $h(z, w)=\frac{H^{n}(x, y)}{s(x) \overline{s(y)}}$ and $\tilde{h}(z, w)=\frac{\tilde{H}^{n}(z, w)}{s(z) \overline{s(w)}}$. Let $s_{i}, i=1, \ldots, n$, be an orthonormal basis for $M$ and $\tilde{s}_{i}, i \geq 1$, an orthonormal basis for $\tilde{M}$. Let $f_{i}=\frac{s_{i}}{s}$ and $\tilde{f}_{i}=\frac{\tilde{s}_{i}}{s}$. From the assumption on the universal covering, we know that $\sum_{i}\left|\tilde{f}_{i}(x)-\tilde{f}_{i}(y)\right|^{2} \geq c$ for some fixed contant $c$ determined by $c_{3}$ and $c_{4}$. By the earlier lemma on the estimate of the difference betweeen the Bergman kernel of $M$ and $\tilde{M}$, we know that

$$
\begin{aligned}
& \sum_{i}\left|f_{i}(x)-f_{i}(y)\right|^{2} \\
= & h(x, x)-h(y, x)-h(x, y)+h(y, y) \\
\geq & \tilde{h}(x, x)-\tilde{h}(y, x)-\tilde{h}(x, y)+\tilde{h}(y, y)-4 C \epsilon \\
= & \sum_{i}\left|\tilde{f}_{i}(x)-\tilde{f}_{i}(y)\right|^{2}-4 C \epsilon \\
\geq & c-4 C \epsilon
\end{aligned}
$$

Hence if the radius of $M$ is sufficiently large so that $\epsilon$ is small, as before, we conclude that $\sum_{i}\left|f_{i}(x)-f_{i}(y)\right|^{2} \neq 0$ and hence the canonical section of $M$ separates $x$ and $y$. This concludes the proof of Lemma 6 .

The condition of Lemma 6 on the existence of a holomorphic section $s \in$ $\Gamma^{(2)}(\tilde{M}, K)$ satisfying $\|s\|_{L^{2}}=1, s(x)=0,\|s(y)\| \geq \kappa$ is satisfied for Hermitian symmetric manifolds. In fact, it suffices to consider bounded linear holomorphic functions.

This concludes the proof of Lemma 5 and hence the proof of Theorem 2 .

\section{EXAmPLES}

Let $M$ be a locally Hermitian symmetric space. It is well-known that the fundamental group $\Gamma$ of $M$ is residually finite in the sense that there is a tower of normal subgroups $\Gamma_{1}=\Gamma, \Gamma_{i}>\Gamma_{i+1}$ and $\bigcap_{i=1}^{\infty} \Gamma_{i}=\infty$. Then $M_{l}=\tilde{M} / \Gamma_{l}$ is a $\left[\Gamma, \Gamma_{l}\right]$-sheeted covering of $M$. In this section, we would like to discuss the effective estimate of $\left[\Gamma, \Gamma_{l}\right]$ to guarantee the very ampleness of $K_{M_{l}}$. To apply results in earlier sections, we are going to consider $M$ to be $M_{l}$ with $l$ to be estimated, and $M_{o}$ to be the lowest manifold in the tower.

First we consider the arithmetic lattices $\Gamma$ of $S U(n, 1)$ defined by Hermitian quadratic forms. This gives rise to arithmetic complex ball quotients

$$
\Gamma \backslash B_{C}^{n}=S U(n, 1) / S(U(1) \times U(n)) .
$$

Let $k$ be a totally real algebraic number field with places $\sigma_{0}=1, \sigma_{1}, \ldots, \sigma_{m}$. Let $Q$ be an Hermitian quadratic form defined on $C^{n}$ over $k$ of signature $(1, n)$ such that the conjugates of $Q$ by the $\sigma_{i}, i>1$, are all negative definite. Let $\mathcal{Z}$ be the ring of integers of $k$ and $G(Q)$ the group of elements in $G L(n+1)$ preserving $Q$. An arithmetic lattice $\Gamma$ of $G=S U(n, 1)$ arising from $Q$ is defined to be a lattice commensurable with $G(Q, \mathcal{Z})=G L(n+1, \mathcal{Z}) \cap G(Q)$, the group of units 
of $Q$. We can diagonalize $Q$ by an element $g \in G L(n+1, k)$ such that $G(Q, \mathcal{Z})$ is commensurable with $G\left(Q^{g}, \mathcal{Z}\right)$. Hence for our purpose, it suffices to consider $\Gamma$ to be $G(Q, \mathcal{Z})$ for some diagonal $Q$ of the form

$$
l_{o}\left|x_{0}\right|^{2}-l_{1}\left|x_{1}\right|^{2}-\cdots-l_{n}\left|x_{n}\right|^{2},
$$

where $l_{i} \in k$ and $l_{i}>0$. The sublattices are given by $\Gamma_{j}=\Gamma\left(q_{j}\right)=\{\gamma \in \Gamma: \gamma \equiv I$ $\left.\left(\bmod q_{j}\right)\right\}$ for a sequence of nested ideals $q_{j}$ with norm approaching infinity. We would call such a sequence a tower of congruence subgroups. We also recall that if $q=t_{1}^{a_{1}} \cdots t_{s}^{a_{s}}$ is the decomposition of an ideal $q$ into prime ideals and $t_{i}$ lies above a rational prime $p_{i}$ with residue class degree $r_{i}$, the norm of $q$ is given by $|q|=\prod p_{i}^{a_{i} r_{i}}$.

Let $\tau\left(M_{l}\right)$ to be the injectivity radius of $M_{j}$. Lemma 2.2.1 of [Y1] allows us to estimate $\tau\left(M_{l}\right)$ in terms of $\left[\Gamma, \Gamma_{l}\right]$.

Lemma 7 ([Y1], Lemma 2.2.1).

$$
\left.\tau\left(M_{l}\right)\right) \geq c+\frac{2}{(n+1)^{2}-1} \log \left(\left[\Gamma, \Gamma_{l}\right]\right),
$$

for an effective constant $c$ independent of $l$. $c$ depends only on the size of the $l_{i}, i=$ $0, \ldots, n$, in the defining equation of $\Gamma$ and the diameter of $M_{o}$.

We note that $\left[\Gamma, \Gamma_{l}\right]$ is actually the ratio $\frac{v o l\left(M_{l}\right)}{\operatorname{vol}\left(M_{o}\right)}$ and is also the ratio of the degree of $M_{l}$ to the degree of $M_{o}$. Recall that the degree of an algebraic manifold is the number $K_{M}^{n}=\int_{M} c_{1}(K)^{n}$. The ratio $\left[\Gamma, \Gamma_{l}\right]$ gives a lower bound of the norm of $q_{l}$, which in turn controls the lower bound of the injectivity radius of $M_{l}$ from the explicit formula of the distance between two points and elementary arithmetic considerations.

This lemma and the theorem of the last section allow us to conclude the following statements.

Theorem 3. For a tower of lattices from Hermitian quadratic forms of complex balls obtained as above, $\Gamma\left(M_{j}, K_{j}\right)$ is very ample on $M_{j}$ as long as the degree of $M_{j}$ is greater than a certain contant $d$ which is an effective constant depending only on the injectivity radius and diameter of $M_{o}$.

Here the degree of $M_{j}$ is the integral of the top power of the Chern class of the canonical line bundle of $M_{j}$, and is related to the degree of $M_{o}$ by a multiple corresponding to the order of the covering. Note that in applying the theorems of the previous sections, the volume $V_{o}$, of $M_{o}$ can be estimated in terms of the injectivity radius and diameter of $M_{o}$.

Remark. For complex ball quotients, there are examples of non-arithmetic lattices in dimension 2 and 3. However, for higher rank Hermitian symmetric spaces $\tilde{M}$, according to the Margulis Arithmeticity Theorem, every cocompact lattice $\Gamma$ of $\tilde{M}$ is arithmetic, as constructed in $[\mathrm{B}]$. Hence we can consider a tower of congruence subgroups defined similarly to the above case of complex hyperbolic balls by considering a nested sequence of ideals $q_{i}$ in the defining number field of $\Gamma$, and regarding $\Gamma_{j}=\Gamma\left(q_{j}\right)=\left\{\gamma \in \Gamma: \gamma \equiv I\left(\bmod q_{j}\right)\right\}$. In this way, $\tilde{M} / \Gamma_{j}$ gives rise to a tower of normal coverings of $M_{o}$. As we also have an explicit formula for the distance function between two points for each (global) Hermitian symmetric space, the argument of the above lemma, or Lemma 2.2.1 of [Y1], allows us to conclude 
that

$$
\left.\tau\left(M_{l}\right)\right) \geq c+f(n) \log \left(\left[\Gamma, \Gamma_{l}\right]\right)
$$

for some constant $f(n)$ depending on the type of the symmetric space $\tilde{M}$, and $c$ depending on $M_{o}$ as before. Now we conclude that $\Gamma\left(M_{j}, K_{j}\right)$ is very ample on $M_{j}$ as long as the degree of $M_{j}$ is greater than a certain contant $d$ which is an effective constant depending only on the injectivity radius and diameter of $M_{o}$, and the type of the Hermitian symmetric space $\tilde{M}$.

Theorem 4. Suppose $M$ is a compact Hermitian locally symmetric space of noncompact type of rank at least 2. There exists an unramified covering $M^{\prime}$ of $M$ of controllable order of covering such that the canonical line bundle of $M^{\prime}$ is very ample. The order of the covering can be estimated in terms of the defining number field of the lattice of $M$ on its universal covering $\tilde{M}$.

This follows from the previous discussions. Note that the injectivity radius and diameter of $M$ are determined by the lattice.

Finally, we add a few more remarks.

Remarks. 1. The arguments of this article can easily be generalized to statements about estimating the injectivity radius so that the canonical section would generate a certain fixed $k$-jet of the manifold.

2. All the arguments can be applied to Kähler manifolds whose Riemannian sectional curvature is bounded by two negative constants. This follows from the fact that there are a lot of $L^{2}$-holomorphic functions on the universal covering, as explained in [Y2]. However, apart from locally Hermitian symmetric spaces, the only such example that we know of in higher dimension cases are the ones constructed by Mostow and Siu in [MS].

3. One consequence of the argument is that, given a hyperelliptic curve $M$, there is an unramified covering $M^{\prime}$ of $M$ which is not hyperelliptic. Moreover, the order of the covering can be estimated in terms of some geometric data as stated in the theorems above.

\section{REFERENCES}

[A] Atiyah, M. F., Elliptic operators, discrete groups, and von Neumann algebras, Colloque "Analyse et Topologie" en l'Honneur de Henri Cartan (Orsay, 1974), Astérisque No. 32-33, Soc. Math France, Paris, 1976, pp. 43-72. MR 54:8741

[B] Borel, A., Compact Clifford-Klein forms of symmetric spaces. Topology 2 (1963), 111-122. MR 26:3823

[BGM] Berger, M., Gauduchon, P., Mazet, E., Le Spectre d'une Varieté Riemannienne, Springer Lecture Notes in Mathematics No. 194, Springer-Verlag, N.Y., 1971. MR 43:8025

[Do1] Donnelly, H., Asymptotic expansions for the compact quotients of properly discontinuous group actions, Illinois J. Math. 23 (1979), 485-496. MR 80h:58049

[Do2] Donnelly, H., Elliptic operators and covers of Riemannian manifolds, Math. Zeit. 223(1996), 303-308. MR 98a:58163

[HT] Hwang, J.-M. and To, W.-K., On Seshadri constants of canonical bundles of compact complex hyperbolic spaces, Compositio Math. 118 (1999), 203-215. MR 2000i:32034

[Lü] Lück, W., Approximating $L^{2}$-invariants by their finite-dimensional analogues. Geom. Funct. Anal. 4 (1994), 455-481. MR 95g:58234

[MP] Minakshisundaram, S., Pleijel, A., Some properties of the eigenfunctions of the Laplaceoperator on Riemannian manifolds. Canadian J. Math. 1, (1949). 242-256. MR 11:108b

[MS] Mostow, G. D., Siu, Y.-T., A compact Kähler surface of negative curvature not covered by the ball. Ann. of Math. (2) 112 (1980), no. 2, 321-360. MR 82f:53075 
[P] Patodi, V. K., Curvature and the eigenforms of the Laplace operator, J. Diff. Geom. 5(1971) 233-249. MR 45:1201

[Y1] Yeung, S. K., Betti numbers on a tower of coverings, Duke Math. J. 73(1994), 201-226. MR 95e:58182

[Y2] Yeung, S. K., Very ampleness of line bundles and canonical embedding of coverings of manifolds, to appear in Compositio. Math.

Department of Mathematics, Purdue University, West Lafayette, Indiana 47907-1395

E-mail address: yeung@math.purdue.edu

Department of Mathematics, The University of Hong Kong, Hong Kong 